\title{
An 18-Year Old Man with Fever and Headache
}

\author{
Amy Baranoski, MD
}

An 18-year-old man was transferred from an outside hospital for evaluation of recent headache, fevers, and laboratory abnormalities.

The patient had a past medical history significant for attention deficit hyperactivity disorder (ADHD) and had presented to an outside hospital with complaints of fatigue, fever, and headache. He described the headache as frontal, severe, and stabbing in nature without associated neck stiffness, photophobia, or phonophobia. He also reported fevers to $105^{\circ} \mathrm{F}$. He was evaluated one day after the onset of symptoms at a local emergency room where a lumbar puncture was performed. By report of the patient and his mother, the lumbar puncture was negative and he was given a prescription for ketorolac for his headache. The patient went home and continued to have fevers and headache for which he was taking acetaminophen and ketorolac. Two days after his initial discharge from the emergency room he presented to another hospital complaining of sharp epigastric pain without radiation in addition to the headache and fevers. He denied nausea, vomiting, or melena. Laboratory data at that time revealed a creatinine of 4.6 and mildly elevated transaminases and the patient was transferred to this hospital for further evaluation and management.

The patient denied chest pain, cough, dyspnea, sore throat, or dysuria. He reported that he had noticed a "sweat rash" in his groin a few weeks prior to his symptoms that went away after persisting for several days. His past medical history was only significant for ADHD. He denied surgical history. Family history was significant for hypertension in both parents. He reported occasional alcohol use. He had recently graduated from high school and lived in a suburban neighborhood. He had been working as a groundskeeper at an outdoor chlorinated pool. He denied tick bites, recent travel, or sexual activity.

Prior to transfer to this hospital, he was given empiric antibiotic treatment with doxycycline and ceftriaxone and was placed on maintenance intravenous fluids, pantoprazole, and prophylactic dose subcutaneous heparin. His only out-patient medication prior to the onset of his symptoms was methylphenidate (Ritalin $\left.{ }^{\oplus}\right)$.

The temperature was $97.4^{\circ} \mathrm{F}$, the pulse rate was 71 , the blood pressure was $105 / 59$, the respiratory rate was 14 and the oxygen saturation was $98 \%$ on room air. On physical examination, the patient appeared well. His pupils were equal and reactive. His sclera were anicteric in contrast to a previous documented examination. He had no oral lesions. The heart and lung examination was unremarkable. He had a left subclavian central venous catheter in place without erythema or fluctuance at the insertion site. He had normal bowel sounds and his abdomen was soft, mildly distended and non-tender to palpation with no appreciated organomegaly or masses. He had no costovertebral tenderness, lower extremity edema, or neck stiffness. Neurological exam revealed no focal deficits with intact cranial nerves.
The patient's initial lumbar puncture results were a glucose of 69, protein of 29, no red blood cells, and one white blood cell. Urinalysis was positive for protein, blood, and bilirubin, with 1025 WBC per high-powered field (HPF), 5 RBC per HPF, occasional WBC and granular casts. Arterial blood gas was $7.37 / 24 / 66 / 14 / 95 \%$ on 3 liters oxygen via nasal canula. Other laboratory data collected after his transfer to this institution were negative and included HIV, EBV antibody and PCR, CMV, HSV, toxoplasmosis antibodies, lyme antigen, and hepatitis serologies. Multiple sets of blood cultures were negative. Complement levels were normal. Leptospirosis antibody was pending during his hospitalization.

The patient's electrocardiogram was normal except for diffuse $\mathrm{T}$ wave flattening. His chest x-ray showed no infiltrate or effusion. An echocardiogram was significant for mild mitral, tricuspid, and pulmonary valvular regurgitation with an ejection fraction of $50 \%$. A right upper quadrant ultrasound showed that his spleen and liver were top normal in size, but was otherwise not remarkable. A CT scan of his abdomen was only significant for a distended stomach and multiple sub-centimeter lymph nodes.

Table 1. Intial Laboratory Data

\begin{tabular}{ll}
\hline Initial Tests & Result \\
\hline White blood cells & 6.7 \\
\hline WBC & $11.6 \mathrm{~B} / \mathrm{L}$ \\
\hline Neutrophils (segs) & $92 \%$ \\
\hline Bands & $5 \%$ \\
\hline PT & $15.3 \mathrm{secs}$ \\
\hline INR & 1.4 \\
\hline PTT & $35.0 \mathrm{secs}$ \\
\hline Sodium & $134 \mathrm{meq} / \mathrm{L}$ \\
\hline Potassium & $4.3 \mathrm{meq} / \mathrm{L}$ \\
\hline Chloride & $99 \mathrm{meq} / \mathrm{L}$ \\
\hline CO2 & $21 \mathrm{meq} / \mathrm{L}$ \\
\hline BUN & $41 \mathrm{mg} / \mathrm{dL}$ \\
\hline Creatinine & $4.6 \mathrm{mg} / \mathrm{dL}$ \\
\hline Glucose & $97 \mathrm{mg} / \mathrm{dL}$ \\
\hline AST & $48 \mathrm{U} / \mathrm{L}(\mathrm{nl} \mathrm{0-37)}$ \\
\hline ALT & $70 \mathrm{U} / \mathrm{L}(\mathrm{nl} \mathrm{0-40)}$ \\
\hline Alk phos & $193 \mathrm{U} / \mathrm{L}$ \\
\hline Total Bilirubin & $8.3 \mathrm{mg} / \mathrm{dL} \mathrm{(nl} \mathrm{0.1-1.2)}$ \\
\hline Direct Bilirubin & $4.7 \mathrm{mg} / \mathrm{dL}$ \\
\hline Amylase & $159 \mathrm{U} / \mathrm{L}(\mathrm{nl} \mathrm{19-68)}$ \\
\hline Lipase & $77 \mathrm{U} / \mathrm{L}(\mathrm{nl} \mathrm{16-63)}$ \\
\hline Troponin I & $0.10(\mathrm{nl}<0.05)$ \\
\hline Urine Drug Screen & $\mathrm{Negative}$ \\
\hline
\end{tabular}


Doxycycline and ceftriaxone were continued during his hospitalization and he was given supportive treatment with intravenous fluids. His creatinine and liver function tests improved upon admission and returned to baseline values in several days. The patient was discharged home on doxycycline with the presumptive diagnosis of leptospirosis.

After discharge to home, the patient's leptospirosis antibody level came back at 12,800 (Negative $<50$, borderline 50-100, positive $>100)$. This patient was seen for follow up one month after discharge and was feeling well.

\section{Discussion}

Leptospirosis is an illness caused by the organism Leptospira interrogans. This is an aerobic spirochete with 18 or more coils per cell. This organism is usually found in tropical environments, but has a world-wide distribution. The highest incidence of illness is after large precipitation events. The organism is found in mammals; most commonly rodents, cattle, goats, horses, or dogs. Animals can become asymptomatic reservoirs with bacterial shedding in the urine. The organism can survive weeks to months in soil and water. The most common mode of transmission in humans is secondary to exposure to contaminated urine via mucus membranes, conjunctiva, or damaged skin. The leptospires then travel in the bloodstream and are carried to multiple organs, where there is endothelial cell disruption leading to vasculitis and ultimately, organ dysfunction.

The largest risk for exposure to leptospirosis is occupational and includes farmers, ranchers, sewer workers, and military personnel. There is also a risk through recreational exposure such as freshwater swimming and kayaking. In 1998, an outbreak occurred in Illinois among triathlon participants. People can also be infected through pets, rodent exposure, and living in contaminated rainwater catchment areas.

Leptospirosis can generally be divided into two groups. 90\% of patients have a mild, self-limited illness. The severe form of leptospirosis is also known as Weil's Disease. It is characterized by hepatic and renal failure. These patients can also have pulmonary hemorrhage, ARDS, and cardiac arrhythmias. EKG changes can vary from frequent premature ventricular contractions to atrial fibrillation or flutter, or even ventricular tachycardia. The mortality rate in Weil's Disease ranges from 5-40\%.

The illness is characterized by abrupt onset of fevers, headache, and myalgias in the majority of patients. There is usually an acute phase which is a one to three day period of constitutional symptoms. The immune phase then follows. There is a two to thirty day incubation period before onset of symptoms. Approximately $50 \%$ of patients have nausea, vomiting, and diarrhea. Less than a third of infected patients have develop a nonproductive cough. Conjunctival suffusion is present in $>80 \%$ of patient with leptospirosis and should cause a high suspicion for the disease if present. Less common symptoms include arthralgias, bone pain, sore throat, and abdominal pain. Muscle tenderness, especially in the calf and lumbar regions, may be present. Less than $10 \%$ of patients develop a pretibial maculo-papular rash. Other less specific signs such as lymphadenopathy, hepatomegaly, or splenomegaly may be present. Aseptic meningitis is usually not seen in acute illness but may occur in up to $80 \%$ of patients later in the course of illness. The aseptic meningitis is secondary to an immune reaction and not caused by the organism itself.

The laboratory data in leptospirosis is non-specific. The WBC count is generally less than 10,000 B/L. Patient may have mildly elevated transaminases, which are usually less than $200 \mathrm{U} / \mathrm{L}$. Urinalysis reveals mild proteinuria and more than 5 WBC per HPF. Patient may have hematuria or hyaline casts. Cerebrospinal fluid is also non-specific with normal glucose, protein between 50 100 and cell counts less than 500. It is possible to grow leptospires in CSF, blood, or urine, especially if cultured during the first seven to ten days of infection. This is a fastidious organism which usually takes one to two weeks to grow, but can take up to three months in some cases. The organism requires Fletcher's, Ellinghausen's, or polysorbate 80 media in order to grow.

Darkfield microscopy will show spirochetes and can be used to make a presumptive diagnosis. PCR can also be used to diagnose leptospirosis. In addition, there are indirect methods of detection including microscopic agglutination test (MAT), which is a highly sensitive and specific test for leptospirosis antibodies. MAT also provides serotype specific information. There are also ELISA and indirect hemaglutinin assays available. These tests do not provide serotype information.

Mild disease can be treated with doxycycline $100 \mathrm{mg}$ PO bid, ampicillin 500-750 mg PO every six hours, or amoxicillin 500 mg PO every six hours. Moderate to severe disease requires intravenous treatment with penicillin G 1.5 million units every 6 hours or ampicillin 0.5-1 gm every 6 hours.

Leptospirosis can be prevented by immunization of livestock and pets. High-risk workers should wear protective clothing including impermeable boots and gloves. Rodent control is also very important in preventing outbreaks, especially after heavy rains. The CDC recommends chemoprophylaxis for people at increased risk with doxycycline $200 \mathrm{mg}$ weekly beginning 1-2 days before exposure.

\section{References}

1. Everett DE. Leptospirosis. UptoDate version 13.2. Available at www.uptodate.com Accessed October 2005

2. Farr RW. Leptospirosis. CID 1995; 21: 1-8.

3. Katz AR et al. Assessment of the Clinical Presentation and Treatment of 353 Cases of Laboratory Confirmed Leptospirosis in Hawaii, 1974-1998. CID 2001; 33:1834-41.

4. Levett PN. Leptospirosis. Clinical Microbiology Reviews. 2001; 14:296-326.

5. Mandell: Principles and Practice of Infectious Diseases, 5th ed, 2000, Churchill Livingstone, Inc.

6. Morgan J. et al. Outbreak of Leptospirosis among Triathlon Participants and Community Residents in Springfield, Illinois, 1998. CID 2002; 34:1593-9.

5. www.cdc.org 